\title{
An outbreak of infantile gastroenteritis due to E. coli 0142
}

\author{
D. H. KENNEDY, G. H. WALKER, R. J. FALLON, J. F. BOYD, \\ R. J. GROSS, AND B. ROWE
}

From the University Department of Infectious Diseases and the Department of Laboratory Medicine, Ruchill Hospital, and the Pathology Department, Western Infirmary, Glasgow, and the Salmonella and Shigella Reference Laboratory of the Central Public Health Laboratory, Colindale, London

SYNOPSIS Twelve cases of gastroenteritis caused by Escherichia coli 0142K86H6 are described. Ten of these cases were clearly involved in an outbreak of cross infection. The other two cases yielded interesting information on infection with $E$. coli.0142. Five cases, two being fatal, required repeated intravenous infusion, and one further infant required parenteral replacement therapy on a single occasion only. Cross infection occurred at the primary site-a ward partitioned into cubiclesdespite full barrier nursing techniques. Infection spread also to two other wards, and resulted from transfer of latently infected cases. Illness in several infants was protracted and debilitating because of the relapsing nature of the infection. The pathology of the two fatalities is reported briefly.

Escherichia coli $0142 \mathrm{~K} 86 \mathrm{H} 6$ was first isolated from infants with diarrhoea in Indonesia (Ørskov, Ørskov, Rees, and Sahab, 1960), and subsequently from an outbreak of diarrhoea among premature babies in Mexico (Olarte and Ramos-Alvarez, 1965). The first outbreak in the UK associated with this serotype occurred in 1969 in a maternity unit at Paisley, near Glasgow. Subsequent outbreaks occurred in 1970 in a Midlands hospital and also in several hospitals in Scotland. In 1971 further cases occurred in Scotland and several Glasgow hospitals were involved (Rowe and Gross, 1971; Love and Gordon, 1971; Roberts, Shrivastava, Emslie, and Ingham, 1971; Love, Gordon, Gross, and Rowe, 1972). In several outbreaks babies were severely ill. Fatalities occurred, though often in cases complicated by other serious illness.

The outbreak described took place in Ruchill Hospital Department of Infectious Diseases, one of two major units serving the Glasgow conurbation. The average number of admissions is 2000 per annum. Of these, about 350 are infants with diarrhoea, who are nursed mainly in two wards divided into cubicles.

\section{The Outbreak}

The first case, a 3-week-old female infant, was adReceived for publication 5 July 1973. mitted to a cubicle ward (A) on 4 February 1971 with a two-day history of diarrhoea (see table and fig 1). Clinically she was moderately dehydrated and biochemical analysis indicated hyponatraemia. $E$. coli 0142 was isolated from the first stool submitted. During the next seven days she had intermittent diarrhoea, but her general condition did not cause concern. Clinical improvement followed the administration of intravenous fluids which were discontinued twice without anxiety. Improvement on each occasion was maintained for about 24 hours but recurrence of vomiting and increased looseness of stools then necessitated further parenteral feeding. On the ninth day she suddenly deteriorated, becoming extremely apathetic and dehydrated and despite further intravenous therapy, she died on 14 February. There was clinical evidence of paralytic ileus at that point but vomiting and diarrhoea were not prominent.

Case 2 was admitted 36 hours later with a history of diarrhoea for seven days and the first stool after admission yielded $E$. coli 0142 . He was mildly dehydrated but responded promptly to oral feeding with half strength Darrow's solution. Diarrhoea stopped within four days and he was discharged well on 22 February.

Cases $3,4,5$, and 7 would normally have been admitted to open ward B (principally for respiratory 


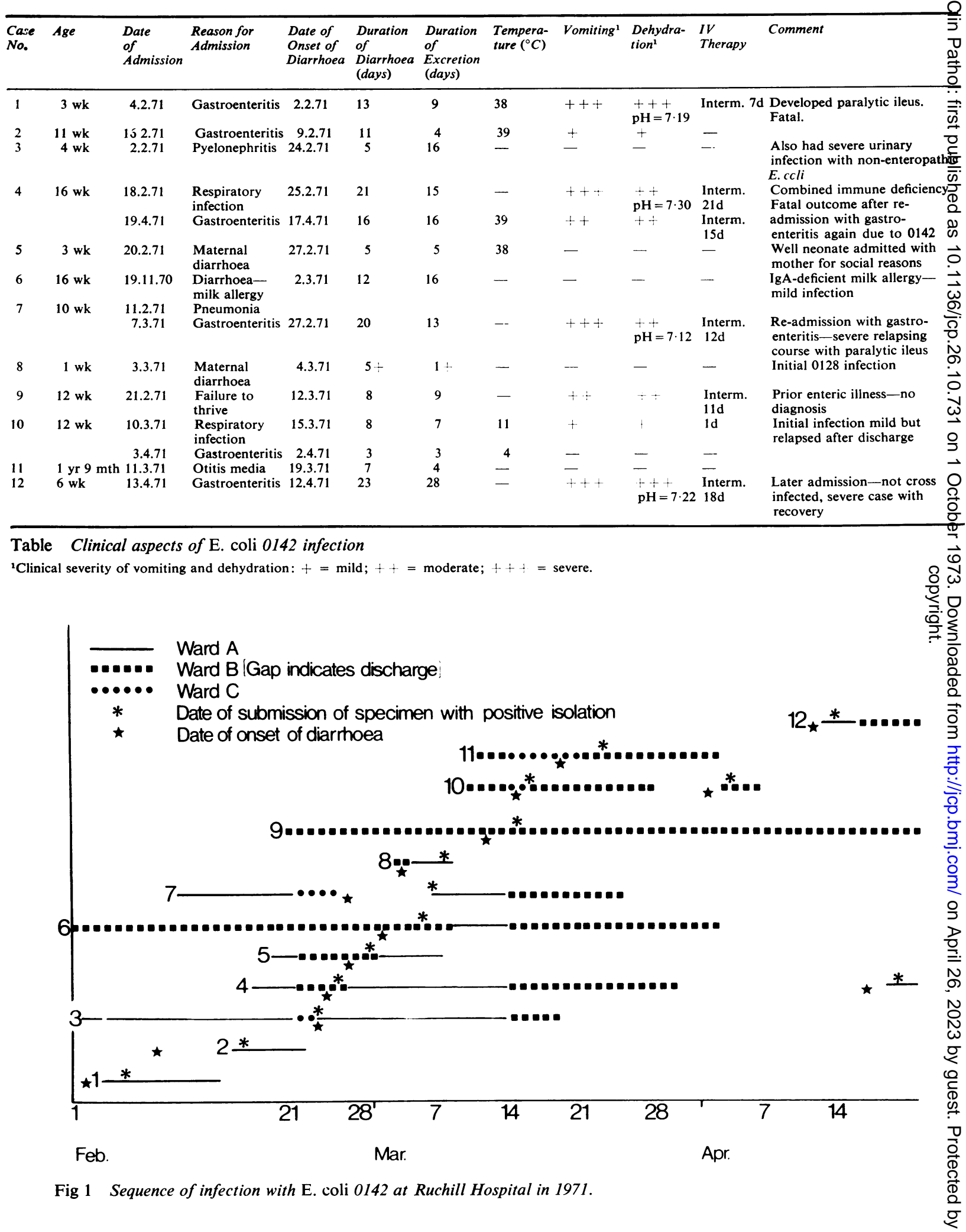


cases), but this ward was full owing to an outbreak of respiratory syncytial virus infection, and they were therefore admitted to ward A. These children did not have diarrhoea and routine admission stool cultures did not reveal the presence of intestinal pathogens. At the time of discharge of case 2 there was considerable demand for cubicle accommodation and therefore cases 4 and 5 were transferred to ward B where accommodation was now available and cases 3 and 7 to another open ward C (see fig 1).

In ward $C$ case 3 developed diarrhoea on 24 February and was returned to ward A. Case 7 was discharged from hospital four days after transfer but developed severe gastroenteritis within two days and was readmitted to ward A. Both infants yielded E. coli 0142 from their stools and it seems likely that cross infection occurred between case 3 and case 7 in ward $\mathrm{C}$ although it is possible that case 7 was infected in ward $A$.

In ward $B$ cases 4 and 5 developed diarrhoea four and six days after transfer and were returned to ward A because $E$. coli 0142 was isolated from their stools. Five of 10 other children in ward B were cross infected from these two cases. Case 6, who was suffering from milk allergy, developed diarrhoea on 2 March. He had been on a milk-free diet for six weeks and had made good progress. Diarrhoea recurred coincidentally with a trial re-introduction of milk feeds. This diarrhoea was believed to be of dietary origin until some days later when a laboratory report indicated that $E$. coli 0142 had been isolated from his stool. He was then transferred to ward A. Case 8, also in ward B, admitted because his mother had postpartum diarrhoea, was found to be excreting E. coli 0128 and he too was transferred to ward A. A stool obtained on discharge four days later subsequently yielded $E$. coli 0142 .

It had been apparent for some time that this organism was highly infectious, but by 14 March it was evident that attempts to prevent cross infection were ineffective, and it was decided therefore to transfer all known and subsequently discovered cases to ward B. Of three remaining infants in this ward, two (cases 10 and 11) who were free from diarrhoea were transferred to ward C. Case 9, who remained in ward $B$, had developed diarrhoea due to E. coli 0142 on 12 March. Cases 10 and 11 were affected one and four days after transfer and hence were returned to ward B. After the end of the outbreak case 12, a 6-week-old infant, was admitted on 13 April with a one-day history of diarrhoea and vomiting. An admission stool yielded $E$. coli 0142 .

E. coli 0142 was not isolated from the stools of any of the staff, and following transfer of known cases to ward B no further instances of cross infection occurred.

Clinically the infection had a wide spectrum of severity. Five cases were mild, the main feature being loose stools. In two cases there was vomiting, diarrhoea, and moderate dehydration which was satisfactorily corrected with half-strength oral Darrow's solution. However, cases 1, 4, 7, 9, and 12 were so ill that they required intravenous therapy on several separate occasions because of prompt relapse on re-introduction of oral feeding. The outstanding biochemical upset was metabolic acidosis which was severe in three cases. Electrolyte disturbance was mild and did not reflect the severe clinical nature of the illness. Only in the two fatal cases and case 12 was there a moderate electrolyte upset. The duration of diarrhoea in three cases was 20 , 21 , and 23 days, during most of which time $E$. coli 0142 was present in the stools. Case 4 was re-admitted on 19 April with a variety of fungal and bacterial infections associated with immune deficiency, probably of the combined type, and continued to excrete $E$. coli 0142 until her death. Case 10 was also found to be excreting $E$. coli 0142 on re-admission.

Four cases were eventually treated with nalidixic acid. Institution of therapy was followed by a steady but not dramatic clinical improvement.

\section{Bacteriological Investigation}

As part of the routine bacteriological examination performed, all faecal specimens were subcultured onto MacConkey agar. Screening for enteropathogenic $E$. coli was carried out by testing 10 colonies and the mass growth by slide agglutination with the following antisera: (1) polyvalent containing antisera for $086,0125,0127,0128,0114$; (2) polyvalent containing antisera for $026,055,0111,0119,0126$, and (3) monovalent antiserum for 0142.

The polyvalent antisera were from commercial sources (Wellcome Reagents Ltd). The monovalent serum for 0142 was produced by the Salmonella and Shigella Reference Laboratory, Colindale.

Colonies giving positive reactions with polyvalent antiserum were tested against the constituent monovalent sera and subcultured into nutrient broth for further examination. Nutrient broth cultures were subcultured on to blood agar and MacConkey agar to check for purity, and were then used for antibiotic sensitivity tests, and also for the preparation of heat-treated suspensions for the confirmation of slide agglutination results by means of tube agglutination tests. All isolates provisionally identified as $E$. coli $\mathrm{O}$ group 142 were sent to the Salmonella and Shigella Reference Laboratory, Colindale, where biochemical identification and full serotyping were 
carried out. Biochemical tests were carried out according to Cowan and Steel (1965) and serological tests according to Kauffmann (1966).

Antibiotic sensitivity tests were carried out using the disc diffusion technique on Oxoid sensitivity test agar plates containing $5 \%$ lysed horse blood. Oxoid Multodisks were used containing the following antibiotics: chloramphenicol $10 \mu \mathrm{g}$, tetracycline $10 \mu \mathrm{g}$, streptomycin $10 \mu \mathrm{g}$, colistin $200 \mu \mathrm{g}$, sulphafurazole $100 \mu \mathrm{g}$, neomycin $30 \mu \mathrm{g}$, nalidixic acid $30 \mu \mathrm{g}$, and ampicillin $2 \mu \mathrm{g}$.

The motile, Gram-negative organisms isolated from the 12 cases described were identified by biochemical and serological tests as E.coli0142K86H6. The biochemical reactions of these isolates were identical and deviated from the usual reactions of $E$. coli only in their failure to produce indole from peptone water media. They were oxidase negative, catalase positive, fermentative, and nitrate reducing. Glucose, lactose, maltose, mannitol, sucrose, dulcitol, arabinose, rhamnose, trehalose, xylose, and raffinose were fermented, and gas was produced from glucose. Salicin, sorbitol, adonitol, inositol, and cellobiose were not fermented. Tests for arginine dihydrolase and ornithine decarboxylase were negative, while that for lysine decarboxylase was positive. Simmon's citrate was not used but Christensen's citrate was utilized after two or three days' incubation. Urea was not broken down. The malonate, phenylpyruvic acid, and gluconate tests were negative. No growth was obtained in potassium cyanide, and hydrogen sulphide was not produced in triple sugar iron agar. The antibiotic resistance pattern of the organisms first isolated from cases 1-12 was as follows: sensitive to chloramphenicol, colomycin, neomycin, nalidixic acid, streptomycin; resistant to tetracycline, sulphafurazole and ampicillin. This resistance pattern is the same as that seen in strains isolated from other outbreaks in the West of Scotland (Love et al, 1972).

\section{Pathology of $E$. coli 0142 Infection}

Necropsy on case 1 was performed 22 hours after death, and showed a dehydrated female infant with dry, wrinkled skin. The tongue, pharynx, and oesophagus were normal. The stomach was collapsed and contained only a small amount of mucus which showed mild 'coffee-grounds' features. The duodenum was normal. The small intestine resembled paralytic ileus being unduly ballooned, and contained an excess of fluid which was partly watery clear and partly curdled milk food. The intestinal wall was thin due to distension and the normal mucosal ridge pattern was virtually obliterated. Some areas were unduly vascular but there was no sign of inflammation. The Peyer's patches were not swollen but four showed bile-staining. The large intestine was collapsed and its scanty contents were semi-fluid. The mucosa showed no inflammation. The liver $(98 \mathrm{~g})$, gallbladder, extra hepatic bile ducts, and pancreas showed no abnormality.

Histologically, a varying degree of autolysis affected the mucosa at all levels of the alimentary tract. Apart from this feature, the stomach showed no abnormality. The jejunum and ileum showed severe blunting of the villi and some oedema of the lamina propria mucosae. At all levels, when conditions were suitable, reparative mucosal changes were seen at the necks of crypts of Lieberkuhn and at the basal attachments of intestinal villi. The mucosal epithelium was undifferentiated at these sites, lacking goblet cells, and in some areas presented the appearances of a simple squamous epithelium with flattened or cubical cells, whereas in other areas the epithelium was rather heaped up resembling a transitional epithelium (fig 2). These features were most pronounced over the bile-stained Peyer's patches, and suggested that prior superficial necrosis and ulceration had taken place. Only in the ileum were there scanty crypt abscesses (figs 3 and 4 ), with intact but dedifferentiated lining mucosal epitheliumo The cell composition in the crypts included neutro

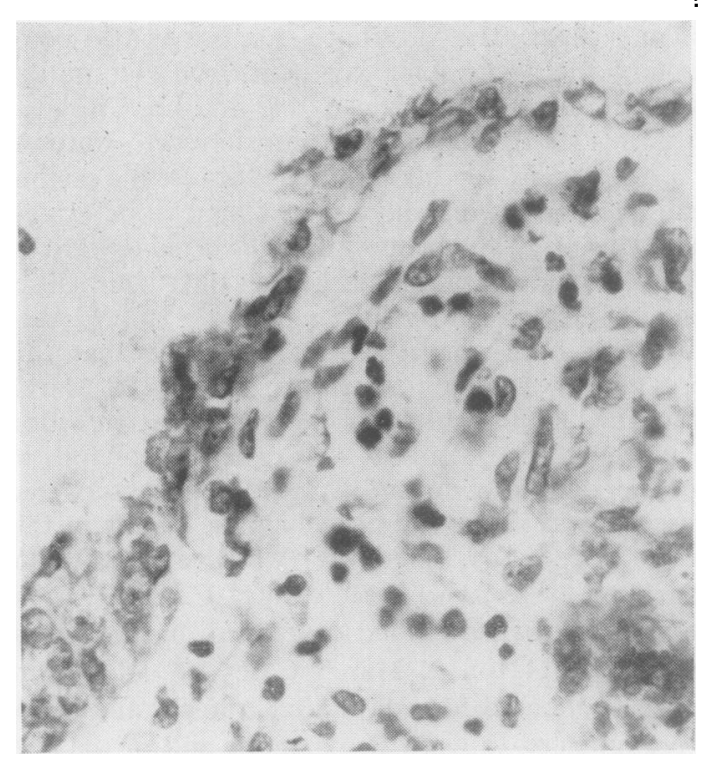

Fig 2 Case 1. Spur between two crypts of Lieberkuhn in small intestine showing mucosal repair by simple rather flattened epithelium at one point, with heaping up to resemble transitional epithelium alongside. Bacteria on surface. Low-grade neutrophil polymorph response in the stroma of the lamina propria. $H \& E \times 512$. 
phil polymorphs, mononuclears, an occasional exfoliated goblet cell, and was sometimes accompanied by a hypersection of mucin. Only in the ileum did the lamina propria show a few aggregations of neutrophil polymorphs, which were also rarely seen in the stroma of blunted villi. The appendix and large intestine were essentially normal, although surface reparative changes affected the mucosa of the former, and one crypt abscess was noted in the ascending colon. The liver showed moderate fatty change and several foci of extramedullary haemopoiesis, which assisted in confirming the immaturity of the infant. The pancreas was normal.

Case 4 was re-admitted to ward $\mathrm{A}$ with gastroenteritis two months after the first admission, and the condition was found again to be associated with E. coli 0142 , ie, exacerbation or re-infection, and she died three weeks later, toxic expidermal necrolysis having developed. Necropsy was performed seven

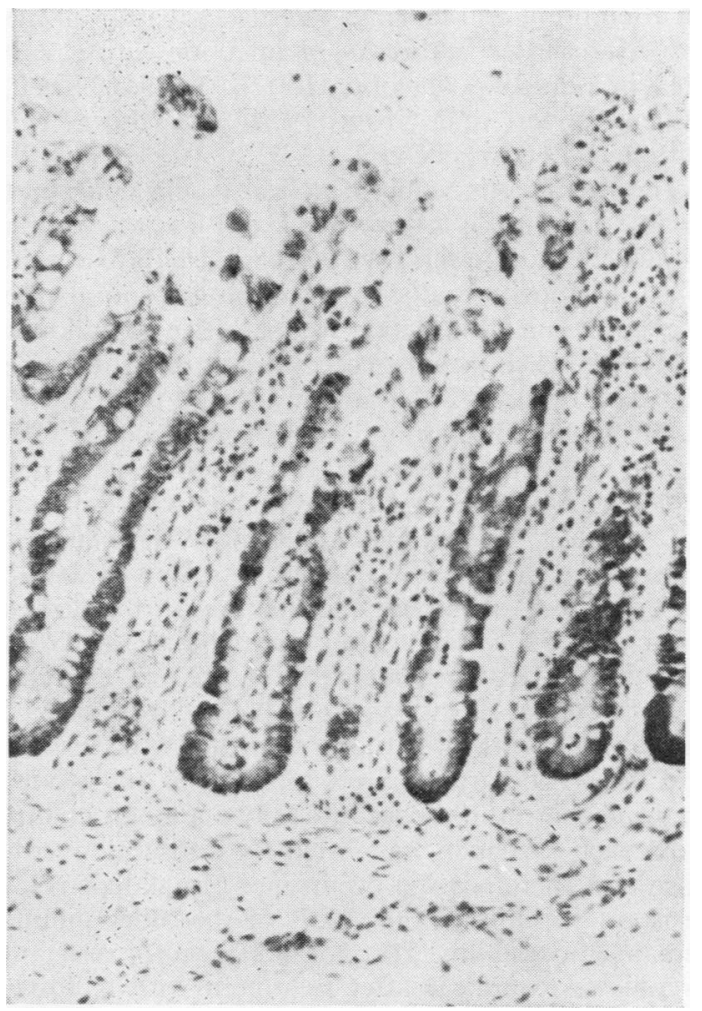

Fig 3 Case 1. Small intestinal mucosa showing stunted villi with oedema of the lamina propria. Collection of neutrophil polymorphs in one lacteal. One mucoid crypt abscess and neutrophil polymorphs in another crypt. Loss of mucosa covering the villi is considered to be due to autolysis. $H \& E \times 128$.

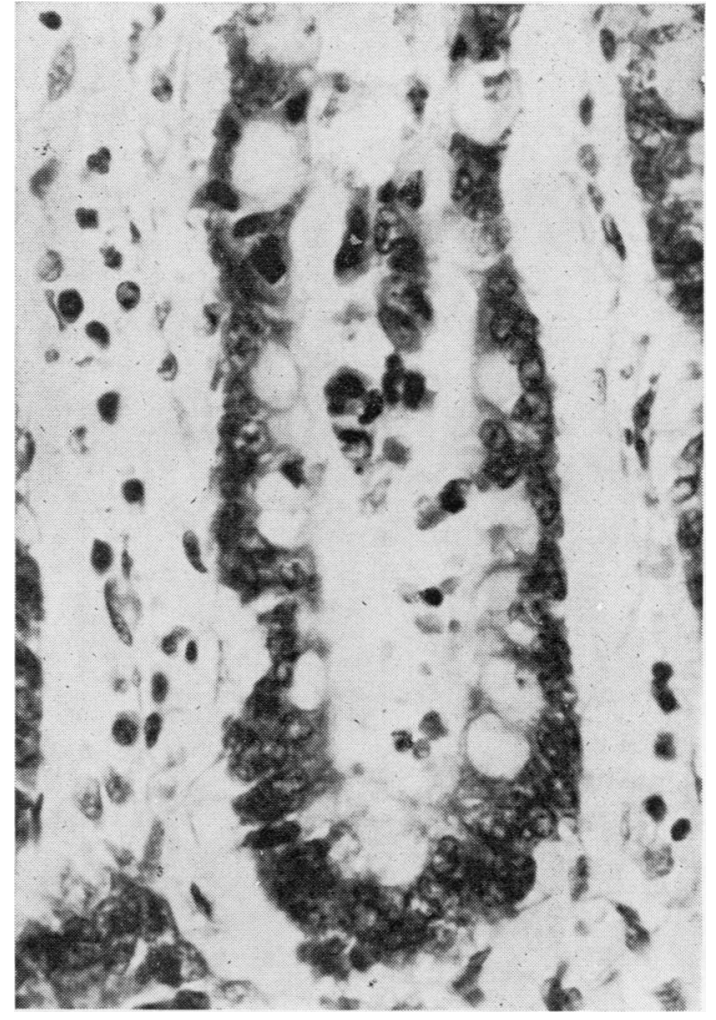

Fig 4 Case 1. Abscess in crypt of Lieberkuhn in small intestine. Mixed cell population. Cells lining the crypt wall are abnormal in appearance, being more flattened than usual. $H \& E \times 512$.

hours after death, and the gross and histological features of the alimentary tract were very similar to those of case 1 (fig 5) except that the Peyer's patches were not bile-stained. 'Blind' selection of blocks to identify Peyer's patches was unsuccessful and this result is likely to represent part of the combined immune deficiency disorder which the infant was considered to have. The appendiceal and large intestinal mucosae were also devoid of lymphoid tissue but were otherwise normal. The liver showed some fatty change, and although the pancreas showed no classical features of mucoviscidosis, all acinar cells were almost totally depleted of zymogen granules.

Neither death showed any histological manifestation of intravascular coagulation such as was reported in all nine deaths of an outbreak due to E. coli 0111 B4 (McKay and Wahle, 1955).

\section{Discussion}

The outbreak caused much concern to the hospital 


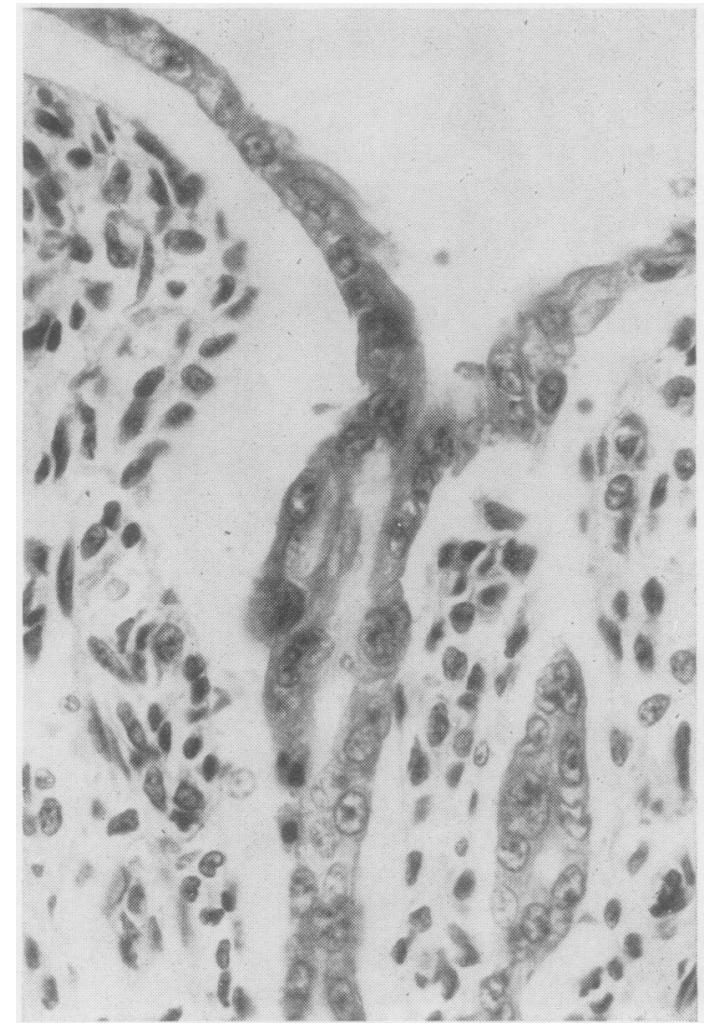

Fig 5 Case 4. Reparative features within crypt of Lieberkuhn and over the surfaces of blunt villi. Nondifferentiated cubical epithelium is evident. $H \& E \times 512$.

staff as in Manchester in 1970 (Ironside, Brennand, Mandal, and Heyworth, 1971), and had a very disruptive effect on the admission potential of the hospital. Clearly the organism involved was highly transmissible but the actual route of infection was not established although all the children were bottle fed with feeds prepared in the ward kitchen. The bottles and teats were disinfected with hypochlorite. An unfortunate aspect of the outbreak was the frequent transfer of patients between wards for the variety of reasons previously described. This underlines the difficulty of maintaining ideal conditions of isolation during a winter period when many children are admitted with respiratory infections. Study of the sequence of cross infection following ward transferrals suggests that there was a minimum interval of three days between probable infection and the occurrence of intestinal upset. The movement of patients between wards during the incubation period of the disease and their subsequent care by different teams of clinicians hindered full and early appreciation of the outbreak which was first recognized by the bacteriology laboratory. The outbreak came to an end when all cases were nursed together in ward $\mathbf{B}$.

Clinically there was a wide variation in the morbidity associated with infection, the severity of the infection being reflected more by severe metabolic acidosis than by disturbance of serum electrolytes.

Age did not seem to play a part in determining response to infection. The long duration of intestinal upset will be seen to be an important feature of the illness, the minimum duration being four days and the maximum being 23 days with a mean of 11 days. The relapsing nature of the illness has been remarked on and it was eventually found necessary to institute intravenous therapy for a prolonged period in some cases. Milk feeding was introduced cautiously, complementary to the parenteral method, only when diarrhoea had been absent for several days. This pattern of a protracted illness, with clinical relapse and recurrence of diarrhoea and vomiting on attempting oral feeding, was also a feature of the two Manchester outbreaks of infection with $E$. coli 0114 in 1968-1969 (Jacobs, Holzel, Wolman, Keen, Miller, Taylor, and Gross, 1970) and with $E$. coli 0119 in 1970 (Ironside et al, 1971).

The therapeutic value of nalidixic acid could not be assessed during this outbreak but it is interesting to note that although case 9 received nalidixic acid in an attempt to eradicate infection he continued to excrete $E$. coli 0142 which was sensitive to nalidixic acid after his course of treatment. Subsequently case 12 was admitted to the same ward and treated with nalidixic acid. The strain of $E$. coli 0142 isolated before treatment was sensitive to nalidixic acid but the isolate after seven days' treatment was resistant. At this stage a repeat faecal specimen was examined from each case, and 10 colonies of $E$. coli 0142 were studied from each specimen. All 10 colonies from case 12 were resistant to nalidixic acid but only one of the 10 colonies from case 9 was resistant. It could be that case 9 was cross-infected from case 12 or that, following his earlier treatment, both sensitive and resistant variants were present in his stools. In the usual routine procedure only one single colony from each specimen was tested for antibiotic resistance and therefore the occurrence of nalidixic acidresistant variants in the stools of the other infants remains a possibility. It is not unknown for resistant organisms to appear after treatment with nalidixic acid (Ronald, Turck, and Petersdorf, 1966). It is of interest that in contradistinction to $E$. coli 0142 , other aerobic Gram-negative flora of both infants remained sensitive to nalidixic acid.

The diagnosis of gastroenteritis in infancy is a very unsatisfactory one at necropsy, unless ante- 
mortem bacteriological support is available. Experience in this hospital has never shown gastritis in such cases when an enteropathogenic $E$. coli has been present, and this observation supports Bray's (1945) suggestion that 'gastroenteritis' is an incorrect term for this condition. Frequently, but not always, the small intestine resembles that of paralytic ileus distension and watery contents are abundant. Yet it is appreciated that this is not paralytic ileus on most occasions since the infant has had pronounced diarrhoea. Agonal intussusceptions are rarely seen. The appendix and large intestine are usually normal, although their contents are not. Grossly, evidence of colitis has never been seen in infancy such as was noted in one of the adult volunteers in the report of Formal, Dupont, Hornick, Snyder, Libonati, and Labrec (1971). Histologically, there is often no abnormality to be noted in the small or large intestinal mucosa, confirming the original observations of Bray (1945) and the later reports of Bray and Beavan (1948), Giles, Sangster, and Smith (1949), and Taylor, Powell, and Wright (1949). Reparative changes similar to those recorded in the two fatal cases of this outbreak have been seen from time to time with E. coli 055 and 0111 (Boyd, unpublished observations), but usually damage is of lesser severity than that described by Rozansky, Berant, Rosenmann, Ben-Ari, and Sterk (1964). The ileum tends to show more changes than the jejunum, an observation that was noted by earlier workers and emphasized again more recently by Rho and Josephson (1967). These observations as well as our own suggest that the condition ought to be well known as acute enteritis of infancy.

The cause of the outbreak was appreciated early because the bacteriology laboratory had a supply of monovalent antiserum to $E$. coli 0142 . This had been provided by the Salmonella and Shigella Reference Laboratory, Colindale, because other outbreaks of infection due to this serotype had been recognized in the west of Scotland (Rowe and Gross, 1971) and all laboratories in the area had been warned of the situation and supplied with a diagnostic antiserum. It is important that strains of $E$. coli from outbreaks of infantile enteritis should be referred to the reference laboratory if they cannot be identified with the sera available to the local laboratory.
We wish to thank Dr I. W. Pinkerton for permission to quote from the clinical records, Mr E. McWilliams for technical assistance, $\mathrm{Mr} \mathrm{P}$. Kerrigan and $\mathrm{Mr} \mathbf{R}$. Ewart, Pathology Department, Western Infirmary, for the illustrations, and the Department of AudioVisual Services, Stobhill Hospital, Glasgow.

\section{References}

Bray, J. (1945). Isolation of antigenetically homogeneous strains of Bact. coli neapolitanum from summer diarrhoea of infants. J. Path. Bact., 57, 239-247.

Bray, J., and Beavan, T. E. D. (1948). Slide agglutination of Bacterium coli var. neopolitanum in summer diarrhoea. J. Path. Bact., 60, 395-401.

Cowan, S. T., and Steel, K. J. (1965). Manual for the identification of Medical Bacteria. Cambridge University Press, Cambridge.

Formal, S. B., Dupont, H. L., Hornick, R., Snyder, M. J., Libonati, J., and Labrec, E. H. (1971). Experimental models in the investigation of the virulence of dysentery bacilli and Escherichia coli. Ann. N.Y. Acad. Sci., 176, 190-196.

Giles, C., Sangster, G., and Smith, J. (1949). Epidemic gastroenteritis in infants in Aberdeen during 1947. Arch. Dis. Childh., 24, 45-53.

Ironside, A. G., Brennand, J., Mandal, B. K., and Heyworth, B. (1971). Cross-infection in infantile gastroenteritis. Arch. Dis. Childh., 46, 815-818.

Jacobs, S. I., Holzel, A., Wolman, B., Keen, J. H., Miller, V., Taylor, J., and Gross, R. J. (1970). Outbreak of infantile gastro-enteritis caused by Escherichia coli 0114. Arch. Dis. Childh., 45, 656-663.

Kauffmann, F. (1966). The Bacteriology of Enterobacteriacae. Munksgaard, Copenhagen.

Love, W. C., and Gordon, A. M. (1971). E. coli 0142 gastroenteritis in Scotland. Lancet, $1,861$.

Love, W. C., Gordon, A. M., Gross, R. J., and Rowe, B. (1972). Infantile gastroenteritis due to Escherichia coli 0142. Lancet, 2, 355-357.

McKay, D. G., and Wahle, G. H., Jr. (1955). Epidemic gastroenteritis due to Escherichia coli 0111 B4. Arch. Path., 60, 679-693.

Olarte, J., and Ramos-Alvarez, M. (1965). Epidemic diarrhea in premature infants. Etiological significance of a newly recognised type of Escherichia coli (0142:K86(B):H6). Amer.J. Dis. Child., 109, 436-438.

Ørskov, F., Ørskov, I., Rees, T. A., and Sahab, K. (1960). Two new E. coli.O-antigens: 0141 and 0142 and two new coli $K$-antigens: K85 and K86. Acta path. microbiol. scand., 48, 48-50.

Rho, Y-M., and Josephson, J. E. (1967). Epidemic enteropathogenic E. coli. Newfoundland, 1963. Canad. med. ass. J., 96, 392-398.

Roberts, W., Shrivastava, S. C., Emslie, J. A. N., and Ingham, H. R. (1971). E. coli 0142 and infantile enteritis in Scotland. Lancet, 1, 757-758.

Ronald, A. R., Turck, M., and Petersdorf, R. G. (1966). A critical evaluation of nalidixic acid in urinary tract infection. New Engl. J. Med., 275, 1081-1089.

Rowe, B., and Gross, R. J. (1971). E. coli 0142 and infantile enteritis in Scotland. Lancet, 1, 649-650.

Rozansky, R., Berant, M., Rosenmann, E., Ben-Ari, Y., and Sterk, V. V. (1964). Enteropathogenic Escherichia coli infections in infants during the period from 1957 to 1962. J. Pediat., 64, 521-527.

Taylor, J., Powell, B. W., and Wright, J. (1949). Infantile diarrhoea and vomiting. Brit. med. J., 2, 117-125. 